\title{
Science in the solar system
}

\section{... and Dr Alan Johnstone, of the Mullard Space Science Laboratories, poses some of still-unanswered questions about the solar system.}

Within the consultative structure of both the European Space Agency (ESA) and the UK Science Research Council, space science is divided between committees at the boundary of the solar system. Although this is a neat arrangement, solar-system science encompasses four almost completely distinct scientific fields, requiring different observational techniques and theoretical approaches. In this article it is not possible to do more than give some idea of the vigour of the research and indicate the principal directions it is taking.

Space plasma physics is concerned with the interaction between charged particles and magnetic fields. The solar wind plasma flowing outward from the Sun forms magnetospheres around the magnetised planets. The magnetospheres contain populations of high temperature plasma confined in the magnetic field.

The particle distributions and the magnetic field configuration of the Earth's magnetosphere are now well-known, but they are not enough to explain the first order operation of the magnetosphere as a plasma machine. The solar wind not only forms the magnetosphere; it is also its principal energy source. The energy is accumulated in the magnetic configuration of the magnetosphere and then released in an explosive event called the magnetospheric substorm, whose most obvious manifestation is the polar aurora. There are many unanswered questions about this process. How is the energy of the solar wind coupled into the magnetosphere and what controls the rate? How is the energy stored and what triggers its release?

The interpretation of satellite observations in such a dynamic system is hampered by the ambiguity between time variations of the whole system and spatial variations along the satellite orbit. The problem can be overcome by deploying a number of satellites in carefully coordinated orbits. This approach has been taken by the ISEE project which has one satellite stationed permanently in the solar wind and a pair of satellites moving close together through the outer magnetosphere to investigate the interaction between the solar wind and the magnetosphere. With the GEOS satellite in geostationary orbit they form a powerful combination to study magnetospheric dynamics.

Another fruitful approach is the comparison of solar system magnetospheres. Each one is dominated by a different effect: Jupiter by its rapid rotation; Mercury by the greater solar wind strength near the sun; and Mars by its atmosphere. The Earth's magnetosphere contains the elements in a dynamic balance. There will be an increase in the use of the plasma in the magnetosphere for plasma physics experiments, especially from Spacelab. The advantages include stable, fully ionised plasma, with no walls to influence the experiment.

The second field is the study of planetary atmospheres. There are now routine observations of the Earth's lower atmosphere for meteorological purposes; and there have been continuing investigations in the upper atmosphere in the regions accessible to satellites. But there have been relatively few observations of the stratosphere and mesosphere in between. A major thrust of atmospheric research is now being directed at the atmosphere between $10 \mathrm{~km}$ and $100 \mathrm{~km}$ through the international Middle Atmosphere Programme. Our ignorance of this part of the atmosphere became apparent when questions were raised about the influence of various pollutants-such as Freon from aerosol cans and the exhaust of high-flying aircraft-on the ozone layer.

The chemical reactions which control the composition of the middle atmosphere are extremely complex. Much more information is required about the spatial distribution of various chemical species, the temperature structure, winds, waves and tidal motions before the controlling processes can be understood. All the available platforms will be used in this essentially exploratory work: aircraft, balloons and sounding rockets for in-situ measurements; and free-flying satellites and Spacelab to make global surveys by remotesensing techniques.

As with magnetospheres, investigating the atmospheres of the other planets gives insight into the origin and evolution of the Earth's atmosphere as well. The two Pioneer Venus space probes will soon be returning detailed information about the dense and hostile Venusian atmosphere.

Solar physicists have made substantial use of space vehicles to study radiation from the Sun at wavelengths from UV to X-ray which do not penetrate through the atmosphere. Their next major project is NASA's Solar Maximum Mission, with 2 European instruments, which is designed to study solar flares during the next maximum of sunspot activity.

The scientific problems are similar to those of magnetospheric substorms. Energy is stored in the magnetic field of Sunspots and then suddenly released in the acceleration of charged particles. Similar questions need answering: how is the flare triggered, and how is the energy transferred to the particles? Since both flares and substorms seem to belong to the same class of plasma processes, any advance in one could assist the other and could also be relevant to other astrophysical and laboratory plasmas.

A second mission, the joint ESA/NASA Solar Polar Probe is going to look at the Sun from a different angle and study the three-dimensional structure of the solar wind. Two spacecraft will use the gravitational field of Jupiter to swing into heliocentric orbits normal to the ecliptic plane taking them over the north and south poles of the Sun respectively.

Finally, the field which has given some of the most spectacular results is the exploration of the planets themselves. US spacecraft are now en-route to Venus, Jupiter, Saturn and Uranus. So far the only European involvement has been by individual scientists but there are projects being discussed now which will be open for possible European participation.

I began by suggesting that the four disciplines within solar system science were grouped together just for administrative convenience. It is true that so far there has been little interaction from them. That may be because the links which must exist have not been fully recognised, but there is an extremely important unifying theme. Each field is concerned with one aspect of the physical environment which makes life possible here on Earth. Why has life developed here and apparently not on the other planets? Is there an important balance which we could unwittingly upset? The tremendous progress that has been made towards understanding all aspects of the origin and evolution of the solar system in the twenty years of research in space science, and the simultaneous realisation that human activities can have damaging effects on a global scale makes it clear that it is a field of research that we ignore at our peril. 